\title{
LEVANTAMENTO DOS MACROINVERTEBRADOS AQUÁTICOS DO CÓRREGO LAGOA SERENA, INSTITUTO DE BIOTECNOLOGIA, UNIARA: AVALIAÇÃO DO POSSÍVEL IMPACTO AMBIENTAL DO REPRESAMENTO
}

PINTO, Alessandra dos Santos; MOURA, Daniela Aparecida; LIMA, Flávia Pâmela Alves de. Alunas de graduação do curso de Ciências Biológicas do Centro Universitário de Araraquara - Uniara, Araraquara, São

Paulo, Brasil. E-mail: alessandrasp@bol.com.br.

CORBI, Juliano José. Programa de Mestrado em Desenvolvimento Regional e Meio Ambiente do Centro Universitário de Araraquara - Uniara, Araraquara-SP. Departamento de Hidrobiologia da Universidade Federal de São Carlos, São Carlos, SP.

\begin{abstract}
RESUMO
O represamento de um rio ou córrego é um dos fatores que têm alterado os ecossistemas aquáticos. Um de seus impactos é a mudança do ambiente lótico para lêntico, influenciando a estrutura e o funcionamento desse ambiente e, consequientemente, modificando a fauna aquática. Entre os indicadores biológicos mais utilizados na avaliação de ecossistemas aquáticos, encontram-se os macroinvertebrados aquáticos, pois refletem as mudanças ocorridas no ambiente além de participarem da ciclagem de nutrientes e do fluxo de energia. $\mathrm{O}$ objetivo deste trabalho foi realizar um levantamento da fauna de macroinvertebrados de um córrego localizado no Instituto de Biotecnologia - Ibiotec do Centro Universitário de Araraquara - Uniara e avaliar o possível impacto ambiental causado pelo seu represamento. Realizaram-se duas coletas em dois pontos diferentes, um a montante e outro a jusante do represamento, onde os macroinvertebrados foram coletados utilizando-se rede em "D", pelo método de varredura, e identificados utilizando-se literatura específica. Foram identificados 289 organismos pertencentes a 15 táxons de macroinvertebrados. Foram calculados os índices de diversidade (Shannon), riqueza de espécies (Margalef) e a dominância de espécies No Ponto 1, encontrou-se uma baixa diversidade de organismos, porém com uma grande abundância de organismos, com predominância da família Chironomidae. No Ponto 2, encontrou-se maior diversidade, porém uma baixa abundância, coma predominância da classe Oligochaeta. Aparentemente, o represamento não influenciou a diversidade faunística, porém, a pequena faixa de mata ciliar e a presença de cultura de cana-de-açúcar próxima ao local parecem estar influenciando a riqueza e a diversidade dos organismos.
\end{abstract}

Palavras-chave: Represa; Bioindicadores; Macroinvertebrados aquáticos.

\begin{abstract}
The construction of reservoirs in streams or rivers has altered the aquatic ecosystems. One of these impacts is the change from lotic to lentic systems, influencing the structure and the functioning of this environment and, consequently, modifying the aquatic fauna. Aquatic macroinvertebrates are the most commonly biological indicators in the assessment of aquatic ecosystems, because they reflect the environmental changes and participate in nutrient cycling and energy flow. The aim of this study was to analyze the macroinvertebrates community of a stream located in the Institute of Biotechnology (Ibiotec-Uniara) and to evaluate the possible environmental impact of damming. Two samples were collected from two different sites, downstream and upward the dam where the macroinvertebrates were collected, using a "D" aquatic net, by the sweeping method, and were identified using specific literature. A total of 289 organisms were identified belonging to 15 taxons of macroinvertebrates. The diversity index (Shannon), species richness (Margalef), and dominance index were calculated. In site 1, we found a low species diversity, but with a high abundance of organisms, with the
\end{abstract}


dominance of the Chironomidae family. In site 2, we found more diversity, but low abundance, with the predominance of the Oligochaeta. Apparently, the impoundment has not influenced the diversity of the aquatic fauna, however, the low diversity of riparian vegetation observed in the sites and the presence of the sugar-cane culture in the adjacent areas could be influencing the richness and the diversity of the organisms.

Keywords: Reservoir; Bioindicators; Aquatic macroinvertebrates.

\section{INTRODUÇÃo}

Reservatórios são sistemas abertos que fazem parte de um sistema maior, denominado bacia hidrográfica (ODUM, 1988). O processo de represamento de um rio consiste em barrar seu escoamento natural, aumentando sua planície de inundação de forma a captar o excedente de água para algum uso humano. Trata-se de um processo comum e amplamente empregado no Brasil, seja com objetivos de geração de energia ou para o abastecimento de água, doméstico e industrial, navegação, irrigação e recreação (BORGES; ROMERO, 2009).

Os processos ecológicos nesses ecossistemas são muito mais complexos e variáveis do que aqueles encontrados em lagos naturais (AGOSTINHO; GOMES, 1997). Em contraposição ao rio, a condição de represa provoca alterações no ecossistema aquático, causando modificações na composição química do sedimento, na água, na circulação e na organização das comunidades biológicas, além de influenciar o rio e suas comunidades bióticas a jusante (SURIANI et al., 2007), sendo que o principal impacto é decorrente da alteração de ambiente lótico para lêntico, que influencia a distribuição da fauna aquática (AGOSTINHO; PELICICE; GOMES, 2008). Segundo Ogbeibu e Oribhabor (2002), as alterações na estrutura da comunidade zoobentônica provenientes da construção de barragens, estão relacionadas a uma redução na riqueza, na abundância e na diversidade na região da barragem e a jusante, em comparação com a região a montante.

Dentro das comunidades aquáticas, os macroinvertebrados representam um dos grupos mais afetados pela construção de reservatórios (HENRY, 1999). Sua distribuição está diretamente relacionada à disponibilidade de alimento e quantidade, tipo de sedimento (orgânica, areia, argila), o substrato (pedra, madeira, macrófitas aquáticas) e qualidade da água (temperatura, oxigênio e substâncias dissolvidas) (CALLISTO, 2000).

Através de estudos com macroinvertebrados em represas, é possível analisar o estado de eutrofização e contaminação do corpo d'água, seu grau de pureza para o consumo humano e animal, sua aceitabilidade para irrigação, usos industriais, piscicultura e demais atividades humanas relacionadas aos recursos hídricos. A comunidade de macroinvertebrados bentônicos é um importante componente do sedimento de rios e lagos, sendo fundamental para a dinâmica de nutrientes, a transformação de matéria e o fluxo de energia (CALLISTO; ESTEVES, 1995). Os invertebrados compreendem o maior número de indivíduos, espécies e biomassa em quaisquer ambientes dulcícolas; entre eles se destacam os insetos, que dominam os sistemas de água doce, quer sob o ponto de vista numérico, como sob a questão relativa à diversidade, podendo ser ultrapassados apenas pelos nemátodos em termos numéricos e de biomassa (WARD, 1992). Os crustáceos e moluscos podem ser abundantes, mas raramente se apresentam em grande diversidade (GULLAM; CRANSTON, 1996). Por essas características peculiares, os invertebrados aquáticos são bons sensores de alterações ambientais e, por isso, têm sido cada vez mais utilizados como bioindicadores da qualidade de água (CORBI; TRIVINHO-STRIXINO, 2006; LOT, 2006).

Os organismos bioindicadores respondem a alterações ambientais por meio de reações comportamentais ou metabólicas mensuráveis, que indicam e refletem alguma mudança no ambiente onde eles vivem (ANDRÉA, 2008). Os macroinvertebrados diferem entre si, em relação à poluição orgânica, desde organismos típicos de ambientes limpos e de boa qualidade de águas (como 
ninfas de Plecoptera e larvas de Trichoptera - Insecta), passando por organismos tolerantes (alguns Heteroptera e Odonata - Insecta e Amphipoda - Crustacea), até organismos considerados mais resistentes (Chironomidae - Diptera, Insecta e Oligochaeta - Annelida) (CALLISTO; MORETTI; GOULART, 2001).

Dentre as características que tornam esses organismos eficazes neste tipo de estudo se destacam: o tamanho relativamente grande, sendo visível a observação direta; em razão da abundância e diversidade de organismos há uma infinita gama de tolerância a diferentes parâmetros de contaminação; o fato de estarem intimamente associados ao substrato os deixa expostos à ações de alterações ambientais; apresentam uma vantagem de refletir as condições existentes antes da coleta de amostras, enquanto os métodos tradicionais oferecem somente a característica da água do momento da coleta. Uma considerável desvantageméo fato de existirem muitos representantes de macroinvertebrados de diversos grupos taxonômicos, surgindo problemas relativos à identificação dos organismos, sendo muitas vezes impossível chegar até o nível de espécie.

Nesse contexto, o presente estudo tem como objetivo o levantamento da comunidade de macroinvertebrados aquáticos, para identificação do possível impacto ambiental causado pelo represamento de um curso d'água, e realizar, através dos resultados obtidos, uma comparação entre os pontos da nascente e jusante da barragem.

\section{Material e métodos}

\section{Área de estudo}

O presente estudo foi realizado no Instituto de Biotecnologia - Ibiotec do Centro Universitário de Araraquara - Uniara, que se localiza na estrada que liga o município de Araraquara-SP ao de distrito Bueno de Andrada-SP. O instituto apresenta uma área total de 16 alqueires, sendo que há uma monocultura de cana-de-açúcar e alguns animais - alguns poucos bovinos. Aárea também possui uma pequena represa, a Lagoa Serena, pertencente à Bacia Hidrográfica do Rio Jacaré-Guaçu (Figura 1).

\section{Caracterização ambiental}

Para o estudo, selecionaram-se dois pontos para comparação: um antes do represamento (Ponto I, Nascente) e o outro após o represamento (Ponto II, Jusante). A nascente, designada como Ponto I, local parcialmente fechado, apresenta vestígios de mata primária e de sucessão ecológica, com predominância de embaúba e samambaia, não apresentando erosão. Apesar de apresentar mata ciliar parcialmente fechada, essa é representada em pequena quantidade e baixa riqueza de espécies.

A jusante da represa foi designada como Ponto II, local considerado como mata aberta. Possui sinais de perturbação ambiental e predominância de plantas exóticas, como: "comigo-ninguém-pode", palmeiras e eucalipto. A mata ciliar é ausente e o terreno considerado como sendo de baixa declividade.

\section{Coleta da fauna}

Foram realizadas duas coletas em cada ponto, sendo uma no mês de março e outra em maio de 2010.

O material foi coletado através do método de varredura, com o auxílio de uma rede em "D" (malha de 0,25 $\mathrm{mm}$ de abertura), por um período de dois minutos, procurando-se os vários biótipos do trecho (áreas de corredeira e remanso), conforme recomendação de Fontoura (1985).

Durante a realização da primeira coleta, foi observado no Ponto II um fluxo constante de água corrente, em razão de a comporta da represa estar aberta, em seu normal funcionamento.

Na segunda coleta, foi observado um fluxo de água reduzido, pois a comporta estava fechada. Entre os períodos das coletas 1 e 2, a represa Lagoa Serena foi parcialmente esvaziada, com o objetivo de facilitar a pesca. Posteriormente, a comporta foi fechada, para que a represa retornasse ao seu normal funcionamento.

\section{Processamento das amostras e identificação dos organismos}

O material coletado nos diferentes pontos foi acondicionado em tambores plásticos que continham água do local. Os tambores foram transportados para 
o laboratório, onde os exemplares foram triados em bandejas de polietileno transiluminadas e os espécimes foram preservados em álcool $70 \%$.

A identificação dos organismos foi feita sob microscópio esteroscópio, com o auxilio de chaves específicas de identificação (MACCAFFERTY, 1981; MUGNAI et al., 2009). Os organismos coletados foram identificados até o nível de família. De acordo com Corbi e Trivinho-Strixino (2006), a avaliação até o nível de família é suficiente para comprovação de impactos ambientais, utilizando macroinvertebrados em corpos d' água localizados em área de Cerrado.

Após a identificação dos espécimes, foram calculadas algumas métricas descritoras da comunidade, tais como: número total de indivíduos, número de famílias, Índice de Diversidade de Shannon (H), Índice de Uniformidade de Pielou (J), riqueza de Margalef (Margalef) e Dominância de táxons. Essas análises foram feitas utilizando-se o programa estatístico PAST (versão 1.68) (Tabela 2). Também foi utilizado o Índice Biótico BMWP e o Índice Biótico Belga (THORNE AND WILLIAMS, 1997).

\section{Resultados E Discussão}

\section{Análise da fauna}

No total, foram identificados 289 organismos pertencentes a 15 táxons de macroinvertebrados. De maneira geral, a classe Insecta foi dominante, com a presença de 6 ordens. A classe Oligochaeta (Annelida) também foi relativamente expressiva (Tabela 1).

$\mathrm{Na}$ primeira coleta foram identificados 124 organismos pertencentes a 6 ordens de Insecta e 13 famílias de macroinvertebrados aquáticos. No Ponto I, foram coletados 105 organismos, pertencentes a 9 famílias, das quais as mais abundantes foram a família Chironomidae (subfamília Tanypodinae), contribuindo com 47,62\% dos organismos, e as famílias Libellulidae contribuindo com 13,33\%, e Helodidae, com 11,43\%. No Ponto II, foram coletados 19 organismos pertencentes a 7 famílias, das quais a família Chironomidae (subfamília Tanypodinae) foi a mais abundante entre os grupos, $\operatorname{com} 26,32 \%$.

$\mathrm{Na}$ segunda coleta foram identificados 165 organismos pertencentes a 5 ordens de Insecta e 11 famílias de macroinvertebrados aquáticos. No Ponto I, foram coletados 154 organismos, pertencentes a 7 famílias. O táxon Chironomidae (subfamília Tanypodinae) foi o mais abundante, contribuindo com $42,86 \%$, a família Helodidae contribuiu com $36,36 \%$, seguida pela família Chironomidae (subfamília Chironominae), com 10,39\%. No Ponto II, foram coletados 11 organismos pertencentes a 4 famílias, das quais a Classe Oligochaeta foi dominante, contribuindo com 54,55\% dos grupos encontrados (Tabela 1).

Os macroinvertebrados aquáticos bioindicadores podem ser classificados de acordo com a sua tolerância em relação aos diferentes fatores ambientais, em três principais grupos: organismos sensíveis, organismos tolerantes e organismos resistentes. O primeiro grupo (sensíveis) engloba principalmente representantes das ordens de insetos aquáticos Ephemeroptera, Trichoptera e Plecoptera, e são caracterizados por organismos que possuem necessidade de elevadas concentrações de oxigênio dissolvido na água (GOULART; CALLISTO, 2003). Neste estudo foram encontrados apenas 3 organismos da ordem Trichoptera (Coleta 1, Ponto II), o que pode indicar boa quantidade de oxigênio neste ponto, provavelmente devido ao fluxo de água contínuo proveniente do ponto de vazão da represa.

De acordo com Goulart e Callisto (2003), o segundo grupo (organismos tolerantes) é formado por uma ampla variedade de insetos aquáticos. Seus principais representantes pertencem às ordens Heteroptera, Odonata e Coleoptera, embora algumas espécies desses grupos sejam habitantes típicos de ambientes não poluídos. Do ponto de vista ecológico, são todos exclusivamente predadores. Nas coletas 1 e 2 esses organismos foram encontrados apenas no Ponto 1. Na coleta 2, ponto I, foi observado um aumento na abundância de organismos pertencentes à família Helodidae, em relação à coleta 1 do mesmo ponto. Segundo os autores, o terceiro grupo é formado por organismos extremamente tolerantes, por isso também podem ser chamados de resistentes. É formado principalmente por larvas de determinados 
gêneros de Chironomidae, outros Diptera e pela classe Oligochaeta. Esses organismos conseguem viver em condição de anóxia (depleção total de oxigênio) por várias horas: muitos são organismos detritívoros, o que favorece a sua adaptação aos mais variados ambientes.

Os Tanypodinae são considerados predadores. Estudos realizados por Beé (2008) indicam que eles se alimentam de algas, fragmentos vegetais e itens de origem animal, principalmente partes de larvas de outros Chironomidae. No presente trabalho, foi encontrada uma grande quantidade de Tanypodinae, em relação aos Chironominae, sendo provavelmente a predação a causa da baixa abundância de Chironominae, organismos que, em ambientes lóticos e lênticos, são encontrados em maior quantidade em relação à quantidade de Tanypodinae.

Organismos da classe Oligochaeta foram encontrados apenas no Ponto 2 das duas coletas: provavelmente são provenientes da área da represa, pois, de acordo com outros estudos, estes organismos são típicos de ambientes lênticos (CORBI; TRIVINHO-STRIXINO, 2002).

O Ponto 1 nas duas coletas demonstrou baixa diversidade de famílias, porém com abundância relativamente grande de indivíduos, predominando a família Chironomidae. Essa baixa diversidade e a ausência de alguns grupos de organismos sensíveis são, possivelmente, causadas pela grande quantidade de matéria orgânica em decomposição, levando à baixa oxigenação da água.

Outros fatores que poderiam influenciar essa baixa diversidade de organismos são: pouca mata ciliar (menos de 30 metros) e a presença da monocultura de cana-de-açúcar, a qual está localizada em uma altitude superior à nascente, recebendo, provavelmente junto com as águas da chuva, fertilizantes e pesticidas. As famílias observadas em ambos os pontos podem estar sofrendo o impacto da aplicação de pesticidas na área, refletido na diversidade e dinâmica das populações. A redução na densidade de populações no Ponto 2 pode ser tanto um efeito direto da contaminação da água, quanto um resultado indireto relacionado às mudanças nas interações entre espécies, à redução das taxas de emergência e reprodução e à menor capacidade de dispersão e colonização na bacia. No Ponto I, também foi possível observar a predominância de táxons predadores (subfamília Tanypodinae e ordens Odonata e Hemiptera).

De um modo geral, ambos os pontos apresentaram baixa diversidade de espécies, com pouca diferença entre eles; desse modo, não há como inferir, até o momento, se o represamento está interferindo na estrutura e no funcionamento do ecossistema. Porém, através da observação do local, foi possível notar que as famílias de espécimes encontradas são condizentes com a situação observada, o que demonstra a importância de áreas verdes e mata ciliar preservadas, para a qualidade da água e, consequentemente, para todo o ecossistema. De acordo com Merrit e Lawson (1992), a produção primária oriunda da mata ciliar é uma importante fonte de alimentação e proporciona diversos habitats para inúmeras espécies aquáticas.

Através dos índices estudados, observou-se na coleta 2 uma relevante diminuição na diversidade e na quantidade de organismos do Ponto 2 em relação à coleta 1, provavelmente ocasionada pelo esvaziamento da represa e posterior fechamento das comportas, que aumentou o fluxo de água e provavelmente deslocou a fauna. Em locais perturbados pelas atividades antrópicas há uma perda ou diminuição de táxons sensíveis e o aumento de táxons tolerantes, o que pode levar à diminuição da diversidade (AZRINA et al., 2006).

Para melhor compreensão dos dados obtidos, foi realizada uma comparação entre o presente estudo e o trabalho de Saulino e Caraccioli (2009), que foi realizado em uma área de preservação permanente no Ribeirão das Anhumas, município de Araraquara-SP. Todos os índices estudados apontaram grandes diferenças em abundância, riqueza e diversidade das famílias, o que ressalta a importância das áreas de preservação permanente para o ecossistema, que proporciona melhorias na qualidade da água e, consequentemente, garante a abundância e diversidade faunística.

O Índice Biótico BMWP apontou excelente 
qualidade de água no estudo de Saulino e Caraccioli (2009), enquanto no presente estudo a qualidade da água nos pontos variou de regular a ruim. $O$ Índice Biótico Belga demonstrou qualidade de água boa (coleta 1, Ponto 1), variando para ruim (coleta 2, Ponto 1); o Ponto 2 variou de ruim (coleta 1) para péssima (coleta 2) - esse mesmo índice apontou para a qualidade da água excelente no Ribeirão das Anhumas.

\section{Conclusão}

Através da análise foi possível observar a baixa riqueza e abundância de táxons em ambos os pontos estudados, revelando que o ecossistema do córrego Lagoa Serena (Ibiotec) se encontra parcialmente impactado e há necessidade de atividades de reflorestamento no local. Os dados mostram que, até o momento, o represamento parece não estar influenciando a estrutura da fauna de macroinvertebrados aquáticos, uma vez que os índices aplicados demonstraram pouca diferença entre os pontos analisados.

\section{Agradecimentos}

Agradecemos ao nosso orientador, Juliano José Corbi, pela sua ajuda e disponibilidade, à coordenadora do curso de Ciências Biológicas, Teresa Muraoka, pelos valiosos conselhos, e à Uniara, pela oportunidade e espaço que nos foram concedidos para a realização deste trabalho.

\section{REFERÊNCIAS}

AGOSTINHO, A.A. GOMES, L.C. Manejo e monitoramento de recursos pesqueiros: perspectivas para o reservatório de Segredo. In: A. A. Agostinho \& L. C. Gomes (eds.) Reservatório de Segredo: bases ecológicas para o manejo. Eduem, Maringá, 387 p. 1997.

AGOSTINHO, A. A.; PELICICE, F. M.; GOMES, L. C. Represamentos e a fauna de peixes neotropicais: impactos e manejo relacionados à diversidade e à pesca. Brazilian Journal of
Biology, vol. 68, 2008. Disponível em: < http:// www.scielo.br/scielo.php?pid=S151969842008000500019\&script=sci_abstract\&tlng=pt $>$ Acesso em: 16 de jul. 2010.

\section{ANDRÉA, M. M. Bioindicadores} ecotoxicológicos de agrotóxicos. Comunicado técnico, Instituto Biológico, 2008. Disponível em: $<$ http://www.biologico.sp.gov.br/ artigos_ok.php?id_artigo=83> Acesso em: 02 set. 2010.

AZRINA, M. et al. Anthropogenic impacts on the distribution and biodiversity of benthic macroinvertebrates and water quality of the Langat River, Peninsular Malaysia. Ecotoxicology and Environmental Safety, 2006.

BATZER, D.P.; RESH, V.H. Macroinvertebrates of a California seasonal wetland and responses to experimental habitat manipulation. Wetlands, 12, 1992.

\section{BEÉ, M.E.T. Ecologia de larvas de} Chironomidae (Diptera) no rio Irani, Santa Catarina, Brasil. Dissertação (Mestrado). Universidade Comunitária Regional de Chapecó, Chapecó, 2008. Disponível em: < http:// www.unochapeco.edu.br/saa/tese/5364/ Dissertacao\%20completa.pdf> Acesso em: 03 set. 2010.

BORGES, J. P.; ROMERO, R. M. Represamento reflexões sobre o seu manejo. Disponível em: <http:// inteligenciaecologica.blogspot.com/2009/09/ represamentos-reflexoes-sobre-o-seu.html $>$ Acesso em: 17 jul. 2010.

CALLISTO, M.; ESTEVES, F.A. Distribuição da comunidade de macroinvertebrados bentônicos em um ecossistema amazônico impactado por rejeito de bauxita - Lago Batata (Pará, Brasil). Oecologia Brasiliensis, Rio de Janeiro, 1995, vol. 1, p. 335- 
348. Disponível em: < http://

www.oecologiaaustralis.org/ojs/index.php/oa/article/ download/17/290> Acesso em: 02 set. 2010.

CALLISTO, M. Macroinvertebrados bentônicos.

In: R. L. Bozelli, F. A. Esteves e F. Roland (ed.), Impacto e recuperação de um ecossitema amazônico. Ed. UFRJ, Rio de Janeiro, p. 141-151, 2000 .

CALLISTO, M; MORETTI, M.; GOULART, M. Macroinvertebrados bentônicos como ferramentas para avaliar a saúde de riachos. Revista Brasileira de Recursos Hídricos. p. 71- 82, 2001.

CALLISTO, M. et al. Biodiversity assessment of benthic macroinvertebrates along a reservoir cascade in the lower São Francisco river (northeastern Brazil). Brazilian Journal of Biology, vol. 65, São Carlos, mai. 2005. Disponível em: <http:// www.scielo.br/scielo.php?script=sci_arttext\&pid= S1519-69842005000200006\&lang=pt $>$ Acesso em: 13 de jul. 2010.

CORBI, J.J.; TRIVINHO-STRIXINO, S. Spatial and bathymetric distribution of the macrobenthic fauna of the Ribeirão das Anhumas reservoir (Américo Brasiliense-SP, Brazil). Acta Limnologica Brasiliensia, 14(1):35-42, 2002.

CORBI, J.J.; TRIVINHO-STRIXINO, S. Influence of taxonomic resolution of stream macroinvertebrate communities on the evaluation of different land uses. Acta Limnologica Brasiliensia, 18(4):469-475, 2006.

FONTOURA, A.P. Manual de vigilância da qualidade das águas superficiais: Avaliação biológica da qualidade da água. Instituto de Zoologia. Faculdade de Ciências - Universidade do Porto, Portugal, 1985.

GOULART, M.D.C.; CALLISTO, M.
Bioindicadores de qualidade de água como ferramenta em estudos de impacto ambiental. Revista FAPAM, ano 2, n. ${ }^{\circ}$ 1, 2003. Disponível em: < http://www.icb.ufmg.br/big/beds/arquivos/ goulartecallisto.pdf $>$ Acesso em: 02 set. 2010.

GULLAN, P. J.; CRANSTON, P. S. The insects: an cutline of entomology. London, 1996. 113p.

HENRY, R. Ecologia dos reservatórios: estrutura, função e aspectos sociais. Botucatu, FAPESPFUNDBIO, 1999. 800p.

LOT, A. Caracterização da qualidade de água do Ribeirão das Cruzes - Araraquara (SP) através de variáveis físicas e químicas e dos macroinvertebrados bentônicos. Tese (Mestrado). Centro Universitário de Araraquara, Araraquara, SP, 2007.

MACCAFFERTY, W.P. Aquatic entomology. Boston: Jones and Bartlett publishers, 1981.

MERRIT, R.W.; LAWSON, D.L. The role of leaf litter macoinvetebrates in stream-floodplain dynamics. Hydrobiologia, 248p, 1992.

MUGNAI, R.; NESSIMIAN, J. L.; BAPTISTA, D.F. Manual de identificação de macroinvertebrados aquáticos do estado do Rio de Janeiro. Ed. Technical Books, 2009.

ODUM, E. P. Ecologia. Ed. Guanabara, Rio de Janeiro, 1988. 434 p.

OGBEIBU, A.E.; ORIBHABOR, B.J. Ecological impact of river impoundment using benthic macroinvertebrates as indicators. Water Research, vol. 36, mai. 2002.

SAULINO, H.L; CARACCIOLI, L. Estudo da riqueza de macroinvertebrados aquáticos de um Área de Preservação Permanente do Ribeirão 
das Anhumas, Araraquara-SP. Trabalho de Conclusão de Curso (Graduação). Centro Universitário de Araraquara, Araraquara, 2009.

SURIANI, A.L.; FRANÇA, R.S.; ROCHA, O. A malacofauna bentônica das represas do médio rio Tietê (São Paulo, Brasil) e uma avaliação ecológica das espécies exóticas invasoras, Melanoides tuberculata (Müller) e Corbicula fluminea (Müller). Revista Brasileira de Zoologia, vol. 24, mar. 2007. Disponívelem: < http://www.scielo.br/ scielo.php?script=sci_arttext\&pid=S0101- 81752007000100003\&lang=pt $>$ Acesso em: 14 jul. 2010.

THORNE, R.ST.J. AND WILLIAMS, W.P. The response of benthic macroinvertebrates to pollution in developing countries: a multimetric system of bioassessment. Freshwater Biology, 37, 671-686, 1997.

WARD, J.V. Aquatic insect ecology 1. Biology and habitat. New York: John Wiley \& Sons, Inc, 1992. $438 \mathrm{p}$.

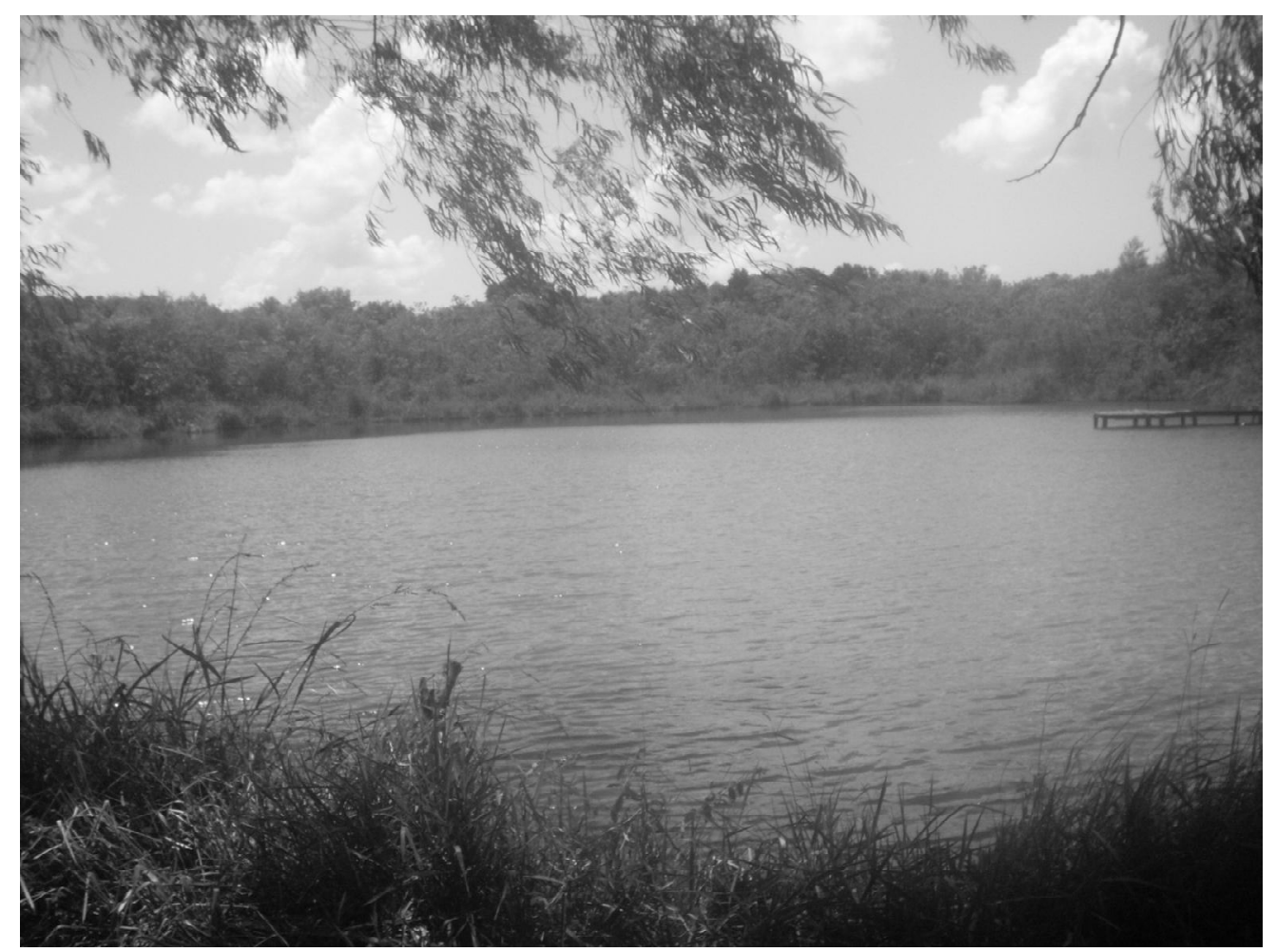

Figura 1 - Represa Lagoa Serena, localizada no Ibiotec. 
Tabela 1 - Total de macroinvertebrados aquáticos coletados no Ponto I e Ponto II do IBIOTEC, município de Araraquara, SP.

\begin{tabular}{|c|c|c|c|c|}
\hline \multirow[b]{2}{*}{ Grupos Taxonômicos } & \multicolumn{2}{|c|}{ COLETA 1} & \multicolumn{2}{|c|}{ COLETA 2} \\
\hline & Ponto I & Ponto II & Ponto I & Ponto II \\
\hline \multicolumn{5}{|l|}{ Diptera } \\
\hline Chironomidae Sub-familia Tanypodinae & 50 & 5 & 66 & 2 \\
\hline Chironomidae Sub-familia Chironominae & 5 & 1 & 16 & 1 \\
\hline Culicidae & - & - & 1 & - \\
\hline \multicolumn{5}{|l|}{ Odonata } \\
\hline Libellulidae & 14 & - & 5 & - \\
\hline Coenagrionidae & 2 & - & - & - \\
\hline \multicolumn{5}{|l|}{ Hemiptera } \\
\hline Belostomatidae & 8 & - & 7 & - \\
\hline Gerridae & 5 & - & 1 & - \\
\hline Naucoridae & 1 & 1 & - & - \\
\hline Veliidae & 7 & 1 & - & - \\
\hline \multicolumn{5}{|l|}{ Coleoptera } \\
\hline Helodidae & 12 & - & 56 & - \\
\hline Dysticidae & - & 3 & - & 1 \\
\hline Gyrinidae & - & - & - & 1 \\
\hline \multicolumn{5}{|l|}{ Lepidoptera } \\
\hline Pyralidae & 1 & - & 2 & - \\
\hline \multicolumn{5}{|l|}{ Trichoptera } \\
\hline Hydropsychidae & - & 3 & - & - \\
\hline \multicolumn{5}{|l|}{ Oligochaeta } \\
\hline Família não identificada & - & 4 & - & 6 \\
\hline \multicolumn{5}{|l|}{ Hirudinea } \\
\hline Glossiphoniidae & - & 1 & - & - \\
\hline Total & 105 & 19 & 154 & 11 \\
\hline
\end{tabular}

Fonte: Dados de pesquisa. 
Tabela 2 - Principais índices quantitativos e qualitativos aplicados a fauna de macroinvertebrados nos dois pontos de coleta, e comparação com o Córrego preservado Ribeirão das Anhumas, Araraquara-SP.

Métricas/Pontos de coleta

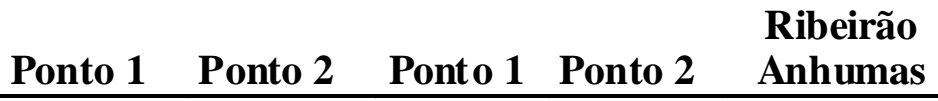

\begin{tabular}{lccccc}
\hline Número de táxons & 9 & 7 & 7 & 4 & 51 \\
Número de indivíduos & 105 & 19 & 154 & 11 & - \\
\% EPT & 0 & 15,79 & 0 & 0 & 19 \\
Índice de Dominância & 0,3183 & 0,2022 & 0,4191 & 0,3884 & - \\
Índice de diversidade de & & & & & \\
Shannon & 1,541 & 1,74 & 1,077 & 1,121 & 2,216 \\
Índice de diversidade de & & & & & \\
Simpson & 0,6817 & 0,7978 & 0,5809 & 0,6116 & - \\
Índice de Riqueza de Margalef & 1,719 & 2,038 & 1,191 & 1,251 & 7,617 \\
Equitabilidade de Pielou & 0,7013 & 0,8941 & 0,5535 & 0,8086 & 0,5635 \\
Índice Biótico BMWP & 44 & 26 & 31 & 12 & 104 \\
Índice Biótico Belga (IBB) & 7 & 5 & 4 & 2 & 10 \\
\hline
\end{tabular}

\begin{tabular}{|c|c|c|c|}
\hline Classe & Faixa de "score" IBB & $\begin{array}{c}\text { Faixa de "score" } \\
\text { BMWP }\end{array}$ & Qualidade da água \\
\hline 1 & $9-10$ & $>81$ & Excelente \\
\hline 2 & $7-8$ & $80-61$ & Boa \\
\hline 3 & 6 & $60-41$ & Regular \\
\hline 4 & $4-5$ & $40-26$ & Ruim \\
\hline 5 & $<3$ & $<25$ & Péssima \\
\hline
\end{tabular}

Fonte: Dados de pesquisa.

RECEBIDO EM 14/10/2010

ACEITO EM 21/12/2010 